\title{
On the Minimum Norm Solution to Weber Problem
}

\author{
Osinuga, I. A. \\ Department of Mathematical Sciences, Olabisi Onabanjo University, Ago-Iwoye Nigeria
}

Bamigbola, O. M.

Department of Mathematics, University of Ilorin, Ilorin, Nigeria

\begin{abstract}
This paper investigates an unconstrained form of classical Weber problem. The main idea is to reformulate Weber problem as an unconstrained minimum norm problem. A result based on the representation of the objective function as a Lipschitzian function, which is necessarily a convex function, is proposed. The existence of global solution to such problem is proven using coercivity assumptions.
\end{abstract}

Keywords: Weber problem, Lipschitzian function, Global Optimization, Coercivity

\section{Introduction}

The continuous single-facility location problem can be stated as follows: find the location for a new facility $X=\left(x_{f}, y_{f}\right)$ such that the sum of the weighted distances from $X$ to $n$ existing facility locations $\left(P_{i}=\left(x_{i}, y_{i}\right), i=1, \ldots n\right)$ is minimized.

If we let $d\left(X, P_{i}\right)$ represent the distance between $X$ and $P_{i}$ and let $w_{i}$ represent the positive weight associated with travel between $P_{i}$ and $X$, then the problem can be formulated as:

$$
\underset{X}{\operatorname{minimize}} f(X)=\sum_{i=1}^{n} w_{i} d\left(X, P_{i}\right)
$$

Where distance is measured using the Euclidean-distance metric i.e.,

$$
d\left(X, P_{i}\right)=\left(\left(x_{f}-x_{i}\right)^{2}+\left(y_{f}-y_{i}\right)^{2}\right)^{1 / 2}
$$

This problem is sometimes called the Weber problem. The Weber problem (WP) has two very important properties. First, $f(X)$ is a convex function which ensures that any local optimum is also a global optimum. Second, the optimal location for the new facility must lie within the convex hull of the existing facility locations.

In recent times, research have been carried out on the minimum norm problems and resolved using several techniques, see $([1,3,4,5,11])$ to mention a few. Such problems have been found useful in approximation theory, statistical estimation problem [11], signal and image reconstruction as well as in other engineering applications [3].

In the [3], the author showed that minimum norm problem can be recast as fixed point problem and showed $x=T x$.He further proved the existence and uniqueness of the minimum solution of operator equation exists and is unique, if $\mathrm{T}$ is non expansive. The research carried out by [4] was to find the minimum norm solution of a linear programs by a Newton-type method which was shown to be globally convergent. In [1], the equivalency of this type of problem was shown using duality principle.

A recent stride reported in [11] was directed at an estimation problem using simple random sampling technique. The idea was used in formulating the estimation problem as an equivalent minimum norm problem in the Hilbert space and resolved by an appropriate application of the classical projection theorem. 
In this paper, we show that a facility location problem otherwise called Weber problem can be recast as a minimum norm problem and resolved as a global solution using coercivity assumptions.

\section{Preliminary and Notation}

Let $X$ be a real Banach space with $\|\cdot\|$ and $X^{*}$ the dual of $X$. For a function $f: X \rightarrow \bar{\Re}$ and $\lambda \in \Re$ consider

$$
\begin{aligned}
& \operatorname{dom} f:=\{x \in X \mid f(x)<+\infty\} \\
& {[f \leq \lambda]:=\{x \in X \mid f(x) \leq \lambda\}} \\
& {[f<\lambda]:=\{x \in X \mid f(x)<\lambda\}}
\end{aligned}
$$

The setdom $f$ is called the domain of the function $f$, while the sets $[f \leq \lambda]$ and $[f<\lambda]$ are the level set and strict level set of $f$ at height $\lambda$. The indicator function of the subset $\mathrm{A}$ of $\mathrm{X}$ is

$$
l_{A}: X \rightarrow \overline{\mathfrak{R}}, \quad l_{A}(\mathrm{x}):=\left\{\begin{array}{lll}
0 & \text { if } & x \in A \\
+\infty & \text { if } & x \in X / A
\end{array}\right.
$$

$\Gamma(X)$ is the class of lower semicontinuous proper convex function $f: X \rightarrow \bar{\Re}$. We conclude the section by introducing some definitions and a theorem (which is proved in [9]) that will be useful subsequently.

\section{Definition 1}

A set is said to be weakly closed (w-closed) if it is closed with respect to the topology $\sigma\left(X, X^{*}\right)$ on $X$.

\section{Definition 2}

A set $K \subset X$ is said to be weakly compact (w-compact) if every sequence from $K$ contains a weakly convergent subsequence.

\section{Definition 3}

A function $f$ is lower semicontinuous (lsc) at $x_{0}$ if for every $\in>0$ there exists a neighbourhood $U$ of $x_{0}$ such that $f(x)>f\left(x_{0}\right)-\in$ for all $x$ in $U$. Equivalently, this can be expressed as

$$
\liminf _{x \rightarrow \infty} f(x) \geq f\left(x_{0}\right)
$$

\section{Definition 4}

A function $f$ is w-lsc if its lower semicontinuous with respect to the topology $\sigma\left(X, X^{*}\right)$ on $X$.

\section{Theorem 1 Weierstrass theorem}

A lower semicontinuous functional on a compact subset $K$ of a normed linear space $X$ achieves a minimum on $K$. 


\section{Unconstrained Minimization Reformulation}

In order to find an unconstrained minimization reformulation for the minimum norm solution for Weber problem (1), we exploit the result of Boyd and Vanderberghe [2] as follows:

As an application, we can think of the points as locations of plants or warehouses of a company, and the links as the routes over which goods must be shipped. The goal is to find locations that minimize the total transportation cost. In another application, the points represent wires that connect pairs of cell. Here the goal might be to place the cells in such a way that the total length of materials needed to interconnect the cells is minimized.

In the simplest version of the problem, the cost $f_{i j}$ associated with arc $(i, j)$ is the distance between nodes $i$ and $j$ : i.e., $f_{i j}\left(x_{i}, x_{j}\right)=\left\|x_{i}-x_{j}\right\|$, such that we minimize

$$
\text { minimize } g(x)=\sum_{(i, j) \in A}\left\|x_{i}-x_{j}\right\|
$$

We can use any norm, but the most commonly used is the Euclidean or the $l_{l}$-norm. We can include nonnegative weights that reflect differences in the cost per unit distance along different arcs:

$$
\sum_{(i, j) \in A}\left\|x_{i}-x_{j}\right\|
$$

By assigning a weight $\quad \mathrm{w}_{\mathrm{ij}}=0$ to pairs of nodes that are not connected and $\mathrm{w}_{\mathrm{ij}}=1$, otherwise, we can express this problem more simply using the objective

$$
\sum_{i<j} w_{i j}\left\|x_{i}-x_{j}\right\|
$$

Thus, the Weber problem (1) can be reformulated as:

$$
\min _{X} f(X)=\min \sum_{i=1}^{n} w_{i} d\left(X, P_{i}\right)=\min \left\|X-P_{i}\right\|
$$

Let $D \subset(X,\|\|$.$) be a non-empty set and consider the distance function$

$$
d_{D}: X \rightarrow \mathfrak{R}, d_{D}(u)=d_{D}(u, c)=\inf \{\|u-c\|: c \in D\}
$$

Having $u \in X$, an important problem consists of determining the set

$$
P_{D}(u)=\left\{c \in D \mid\|\bar{u}-u\|=d_{D}(u)\right\}
$$

$\bar{u} \in P_{D}(x)$ is called a minimum solution of $x$ by elements of $D$.

\section{PROPOSITION 3.1}

Let $d_{D}: D \rightarrow \Re$ be a distance function and $D \subset(X,\|\|$.$) \quad be a non-empty set.$

Then $d_{D}$ is Lipschitzian.

Proof of proposition 3.1

Let $d_{D}(u)=\inf \{\|u-c\|: c \in D\}, d_{D}(y)=\inf \{\|y-c\|: c \in D\}$

$$
d_{D}(u)-d_{D}(y)=\|u-c\|-\|y-c\| \leq\|u-y\|
$$


Using triangle inequality

$$
\begin{aligned}
& \left|d_{D}(u)-d_{D}(y)\right|=\|u-c\|-\|y-c\| \mid \leq\|u-y\| \\
& \Rightarrow\left|d_{D}(u)-d_{D}(y)\right| \leq\|u-y\|
\end{aligned}
$$

For all $u, y \in X$

$\Rightarrow d_{D}$ Is Lipschitzian with Lipschitz constant 1 .

\section{Existence and Uniqueness Theorems}

Consider the constrained

$$
\begin{aligned}
& \min f(x), x \in C \\
& \text { subject to } l_{C}
\end{aligned}
$$

To problem (13), we can associate an unconstrained problem:

$$
\min \bar{f}(x), x \in X
$$

where $\bar{f}=f+l_{C}$

we call value of problem (13) the extended real

$$
v(P)=v(f, C)=\inf \{f(x) \mid x \in C\} \in \mathfrak{R}
$$

An optimal solution of problem (13) is an element $\bar{x} \in C$ with the property that $f(\bar{x})=v(P)$. We denote by $S(P)$ or $S(f, C)$ the set of optimal solutions of problem (13). Therefore,

$S(P)=\{\bar{x} \in C \mid \forall x \in X ; f(\bar{x}) \leq f(x)\}=\{\bar{x} \in X \mid \forall x \in X ; \bar{f}(x) \leq f(x)\}=S(\bar{P})$

if $C \cap \operatorname{dom} f \neq \phi$. The set $S(f, C)$ is denoted by $\operatorname{argmin} f$

The most important result which assures the existence of minimum solution for (1) is the famous Weierstrass's theorem. But we may use for the same purposes, some coercivity conditions because the underlying spaces are not compact. It is obvious that $f$ is coercive if and only if all level set $[f \leq \lambda]$ are bounded; when $f$ is convex then $f$ is coercive if and only if the level set $[f \leq \lambda]$ is bounded for some $\lambda>\inf f$. 


\section{DEFINITION 4.1}

Let $f: X \rightarrow \Re$, we say $f$ is coercive if

$$
\lim _{\|x\| \rightarrow \infty} f(x)=\infty
$$

\section{Lemma 4.1}

Let $(X,\|\cdot\|)$ be a normed space and $f: X \rightarrow \mathfrak{R}$. Then $\lim _{\|x\| \rightarrow \infty} f(x)=\infty$ if and only if $[f \leq \lambda]$ is bounded for every $\lambda \in \mathfrak{R}$ i.e., $f$ is coercive if and only if the level set is bounded.

\section{Proof of Lemma 4.1:}

Since $f$ is coercive i.e. $\lim _{\|x\| \rightarrow \infty} f(x)=\infty, \exists$ a scalar $r>0$ such that $\|x\|>r, f(x)>\lambda \quad \forall \lambda \in \mathfrak{R}$

$$
\Leftrightarrow \lambda \in \mathfrak{R}, \exists r>0: f \leq \lambda \subset B(0, r)
$$

This completes the proof.

\section{Theorem 4.1 ([13, p.100])}

Let $f \in \Gamma(X)$

(i) If there exists $\lambda>v(f, C)$ such that $f \leq \lambda$ is weakly compact, then $S(f, C) \neq \phi$

(ii) If $X$ is a reflexive Banach space and $f$ is coercive then $S(f, C) \neq \phi$

\section{Proof of Theorem 4.1}

(i) Of course, $v(f, X)=v(f,[f \leq \lambda])$. Since $f$ is lower semicontinuous (lsc) and convex, $f$ is weakly lower semicontinuous. The conclusion follows using the Weierstrass theorem applied to the function $\left.f\right|_{[f \leq \lambda]}$

(ii) Because $f$ is coercive (see Lemma 3.1), $[f \leq \lambda]$ is bounded for every $\lambda \in \Re$ since $[f \leq \lambda]$ is weakly closed and $X$ is reflexive, we have that $[f \leq \lambda]$ is weakly compact for every $\lambda \in \mathfrak{R}$. The conclusion follows from (i).

\section{Theorem 4.2}

Let $D \subset X$ be a non-empty closed convex set and $u_{0} \in X$

(i) If $X$ is a reflexive Banach space then $P_{D}\left(u_{0}\right) \neq \phi$

(ii) If $X$ is a strictly convex normed space then $P_{D}\left(u_{0}\right)$ has at most one element.

\section{Proof of Theorem 4.2}

(i) Let us consider the function $f:=\left\|-u_{0}\right\|+l_{D}$. Since $f$ is Lipschitzian, then it is necessarily convex and lower semicontinuous. By theorem 4.1, there exists $\bar{u} \in X$ such that $f(\bar{u}) \leq f(u)$ for every $u \in X$ i.e. $\bar{u} \in P_{D}\left(u_{0}\right)$ 
(ii) We have already seen that $P_{D}(u)=\{\mathrm{u}\}$ for $u \in D$. Let $u \notin D$ suppose that there are two distinct elements $x_{1}, x_{2}$ in $P_{D}(u)$. Then $\frac{1}{2}\left(x_{1}+x_{2}\right) \in D$, and

$$
\begin{aligned}
& \left\|x_{1}-u\right\|=\left\|x_{2}-u\right\|=d_{D}(u)>0 \text {. Since } X \text { is strictly convex, we obtain } \\
& \left\|\frac{1}{2}\left(x_{1}+x_{2}\right)-u\right\|=\left\|\frac{1}{2}\left(x_{1}-u\right)+\frac{1}{2}\left(x_{2}-u\right)\right\|<\frac{1}{2}\left\|x_{1}-u\right\|+\frac{1}{2}\left\|x_{2}-u\right\|=d_{D}(u)
\end{aligned}
$$

This contradiction proves that $P_{D}(u)$ has at most one element.

\section{Concluding Remarks}

In this paper, we investigated and reformulated the Weber problem as an equivalent minimum norm problem. We have also shown that the objective function is Lipschitzian and thus, established the existence of global minimum norm solution using coercivity conditions.

\section{References}

[1] Bamigbola, O. M and Osinuga, I. A (2003). Resolution of Extremization Problems Using Duality Principle. Proceedings African Mathematical Union-International Conference of Mathematical Scientists, University of Agriculture, Abeokuta, Nigeria, 207-213.

[2] Boyd, S. and Vandersberghe, L(2004). Convex Optimization, Cambridge University Press, United Kingdom.

[3] Ferreira, Paulo Jorge S. G(1996). The existence and uniqueness of the minimum norm solution to certain linear and non linear problems. Signal Processing, 55, 137-139.

[4] Kanzow, C., Qi, H. and Qi, L(2000). On the minimum Norm Solution of linear Programs. Applied mathematics Report, AMROO/14, 1-11

[5] Klans, k. and Hannu, S(2005). Minimum Norm Solution for Cooperative Games. Research Report, Department of Economics, University of Turku, Finland, 115,

$1-23$

[6] kreyzig, E (1978). Introductory Functional Analysis with Applications. Wiley, New York.

[7] Kuhn, H. W(1973). A note on Fermat's Problem, Mathematical programming, 4, 98-107

[8] Love, R. F., Morris, J. G. and Wesolowsky, G. O(1988). Facilities Location: Models and methods Elsevier, New York.

[9] Luenberger, D. G(1969). Optimization by vector space methods. Wiley, New York.

[10] Maddox, I. J (1988). Elements of functional Analysis Cambridge University Press, Cambridge.

[11] Oniyide, O. R. and Osinuga, I. A(2006). On the Existence of Best Sample in Simple Random Sampling, ABACUS, 33(2B), 290-294

[12] Stephen, E. B and Cavalier, T. M(1996). An Efficient Algorithm for Facility location in Presence of Forbidden Regions, European Journal of Operational Research, 90, 56-70

[13] Zalinescu, C (2002). Convex Analysis in General Vector Spaces, World Scientific, Singapore. 